\title{
On Bilevel Multi-Follower Decision Making: General Framework and Solutions
}

\author{
Jie Lu, Chenggen Shi, Guangquan Zhang \\ Faculty of Information Technology \\ University of Technology, Sydney \\ Po Box 123, Broadway, NSW 2007, Australia \\ \{jielu, cshi, zhangg\}@it.uts.edu.au
}

\begin{abstract}
Within the framework of any bilevel decision problem, a leader's decision is influenced by the reaction of his or her follower. When multiple followers who may have had a share in decision variables, objectives and constraints are involved in a bilevel decision problem, the leader's decision will be affected, not only by the reactions of these followers, but also by the relationships among these followers. This paper firstly identifies nine different kinds of relationships $\left(S_{1}\right.$ to $\left.S_{9}\right)$ amongst followers by establishing a framework for bilevel multifollower decision problems. For each of the nine a corresponding bilevel multi-follower decision model is then developed. Also, this paper particularly proposes related theories focusing on an uncooperative decision problem (i.e., $S_{l}$ model), as this model is the most basic one for bilevel multi-follower decision problems over the nine kinds of relationships. Moreover, this paper extends the Kuhn-Tucker approach for driving an optimal solution from the uncooperative decision model. Finally, a real-case-study of a road-network problem illustrates the application of the uncooperative decision model and the proposed extended Kuhn-Tucker approach.
\end{abstract}

Keywords: Linear bilevel programming, Bilevel multiple-follower programming, Decisionmaking optimization, Kuhn-Tucker approach, Road network 


\section{Introduction}

Bilevel decision (also called bilevel programming or bilevel optimization) techniques are mainly developed for solving decentralized management problems with decision makers in an hierarchical organization. A decision maker at the upper level is known as the leader, and at the lower level, the follower $[6,7]$. Each decision maker (leader or follower) tries to optimize his/her own objective function with or without considering the objective of the other level, but the decision of each level affects the objective optimization of the other level [4]. Therefore, the leader may be able to influence the behavior of the follower without completely controlling the follower's action. At the same time the leader may be simultaneously affected by the follower's behavior. An hierarchical optimization structure appears naturally in many aspects of resource planning, management and policy making, including water resource management, financial planning, land-use planning, production planning (coordination of multi-divisional firms, network facility location), and transportation planning (network design, trip demand estimation). Amouzegar and Moshirvaziri [1], Bard [4] and Labbe et al. [8] have already recognized the need to consider these planning problems from a bilevel or multi-level modeling point of view.

In general, there are two fundamental issues in both bilevel decision theory and practice.

One is how to model a real-world bilevel decision problem that may have various situations at the two decision levels, and the other is how to find an optimal solution for the decision problem. A number of researchers (e.g., [3, 7, 9]) have established original forms of optimality conditions for bilevel programming. A number of bilevel decision approaches and algorithms $[2,4,5]$ have been proposed to find an optimal solution. This includes the 
most successful Kuhn-Tucker approach [4]. Although much research has been carried out in this area, the existing bilevel technology has been mainly focused on a specific situation comprising one leader and one follower. However, in the case of a real-world bilevel decision problem, the lower level of a bilevel decision may involve multiple decision units. The leader's decision is therefore affected by the objectives and strategies of the multiple followers. For each possible decision of the leader, those followers may have their own, different, reactions. The relationships between these multiple followers can be various. They may or may not share their decision variables. They may have individual objectives and constraints, but work with others cooperatively, or may have common objectives or common constraints. For example, as a leader, the Government's objective in land-use planning is to maximize profiles by establishing some suitable agricultural development policies. Multiple agricultural groups, involving farmers, conservationists, Aboriginal groups, and regions will affect the Government's policy-making in land-use. Each agricultural group, for example, a region, as a follower, has its own individual policies to optimize its objective towards different government policies in land-use. These followers may share the same decision variables, or may have the same objectives or constraints. In such a case, the decision of the Government (the leader) is partially dependent on the 'environment data' put forward by all these agricultural groups (the followers). This is a typical BLMF decision problem.

Following our previous work $[10,11]$, this research generalizes a framework for the BLMF decision problem, which describes nine different kinds of relationship (situations $S_{1}$ to $S_{9}$ ) amongst the followers. Under this framework, for each of the nine a corresponding BLMF decision model is developed. As already shown in this framework, the uncooperative $\left(S_{l}\right)$ 
model is the most popular and the most basic situation for BLMF decision problems. This paper thus presents a definition of an optimal solution and related theories for the $S_{1}$ model. It further extends the Kuhn-Tucker approach for solving the $S_{1}$ model with a real case-study of a road network problem. Related approaches for solving other eight situation models within this framework will be explored further in a separate research paper.

This paper is organized as follows. In Section 2, the framework for BLMF decision problems is proposed. Related theories focusing on the uncooperative $\left(S_{1}\right)$ model of BLMF decision problems are given in Section 3. An extended Kuhn-Tuck approach for solving the $S_{1}$ model is presented in Section 4. A real-case example of a road-network problem for the extended Kuhn-Tucker approach is illustrated in Section 5. Discussions, further remarks, and future research plans are concluded in Section 6.

\section{A Framework for Bilevel Multi-follower Decision Problems}

Different reactions could be generated at the lower level towards each possible action conducted at the upper level when multiple followers are involved in a bilevel decisionmaking. Moreover, different relationships among these followers could cause multiple different processes for deriving an optimal solution for the upper level's decision-making. Therefore, the leader's decision will be affected, not only by the reactions of these followers, but also by the relationships among these followers. Basically, there are three different kinds of relationships among the followers determined by the form of a share in decision variables, the first relationship factor. The first kind of these relationships is the uncooperative situation where there is no sharing of decision variables among the followers. In such a situation, there are obviously neither shared objectives nor shared 
constraints among the followers. The second case is the cooperative situation where the followers totally share the decision variables in their objectives and constraints. However, there are several different sub-cases within the cooperative situation which are determined by the relationships among the objectives (the second relationship factor) and constrains (the third relationship factors) of the followers. Each follower may have an individual objective whatever sharing their constraints with other followers. For example, one agricultural group has its objective to maximize its profile of agriculture, and another agricultural group's objective is to maximize its land sustainability, towards the Government's policy in land-use. The two followers share all other decision variables, but have different objectives. Another pair of sub-cases is that the followers have their common objectives whatever sharing their constraints. For example, for any governmental agricultural policy, the two agriculture groups have their common objective to maximize its profile of agriculture. But they may or may not share constraints in financial, environment protection, and cultural in the context of attempting to achieve an optimal solution. The last case is the partial cooperative situation where the followers partially share decision variables in their objectives or constraints or both. Similar to the second case, four subcases are involved within this one as well.

Based on the three cases and their various sub-cases determined by the three relationship factors, decision variables, objectives and constraints, totally nine different kinds of situations among the followers are identified, named $S_{1}, S_{2}, \ldots, S_{9}$. A framework is established to describe these situations in Table 1. For a bilevel decision problem, if some followers share their decision variables or some not, it will be dealt with as a variable 
sharing situation. Similar, if some followers share their objective (or constraint) functions or some not, it will be dealt with as an objective (or constraint) sharing situation.

Table 1: A framework for the bilevel multi-follower decision problem

\begin{tabular}{|c|c|c|c|c|c|}
\hline \multirow[b]{2}{*}{ Relationships } & \multirow[b]{2}{*}{$\begin{array}{l}\text { Kinds of } \\
\text { relationship }\end{array}$} & \multicolumn{3}{|c|}{ Relationship factors } & \multirow[b]{2}{*}{$\begin{array}{l}\text { Situations } \\
\left(S_{i}\right)\end{array}$} \\
\hline & & $\begin{array}{l}\text { Decision } \\
\text { variables }\end{array}$ & Objectives & Constraints & \\
\hline \multirow{9}{*}{$\begin{array}{l}\text { Among the } \\
\text { followers }\end{array}$} & Uncooperative & Individual & Individual & Individual & $s_{1}$ \\
\hline & \multirow{4}{*}{ Cooperative } & \multirow{4}{*}{ Sharing } & \multirow[t]{2}{*}{ Sharing } & Sharing & $s_{2}$ \\
\hline & & & & Individual & $s_{3}$ \\
\hline & & & \multirow[t]{2}{*}{ Individual } & Sharing & $s_{4}$ \\
\hline & & & & Individual & $s_{5}$ \\
\hline & \multirow{4}{*}{$\begin{array}{l}\text { Partial } \\
\text { cooperative }\end{array}$} & \multirow{4}{*}{$\begin{array}{l}\text { Partial } \\
\text { individual } \\
\text { and } \\
\text { partial } \\
\text { sharing }\end{array}$} & \multirow[t]{2}{*}{ Sharing } & Sharing & $s_{6}$ \\
\hline & & & & Individual & $s_{7}$ \\
\hline & & & \multirow[t]{2}{*}{ Individual } & Sharing & $s_{8}$ \\
\hline & & & & Individual & $s_{9}$ \\
\hline
\end{tabular}

Each situation shown in the framework will require a specific BLMF decision model for describing it, and a specific approach for deriving an optimal solution for the leader's decision. Based on the basic linear bilevel decision problem model $[6,7]$, the decision model of the nine specific situations are established and described respectively as follows. 


\section{$S_{1}$ Model for BLMF decision problems}

For $\quad x \in X \subset R^{n}, \quad y_{i} \in Y_{i} \subset R^{m_{i}}, \quad Y=\left(Y_{1}, Y_{2}, \cdots, Y_{k}\right)^{T}, \quad F: X \times Y_{1} \times \ldots \times Y_{K} \rightarrow R^{1}$, $f_{i}: X \times Y_{i} \rightarrow R^{1}$, and $i=1,2, \ldots, K$, a linear BLMF decision problem in which $K(\geq 2)$ followers are involved and there is no shared decision variable, objective function or constrain function among them is defined as follows (called an uncooperative decision model).

$$
\left\{\begin{array}{c}
\min _{x \in X} \quad F\left(x, y_{1}, \cdots, y_{K}\right)=c x+\sum_{s=1}^{K} d_{s} y_{s} \\
\text { subject to : } A x+\sum_{t=1}^{K} B_{t} y_{t} \leq b
\end{array}\right.
$$

where $y_{i}(i=1,2, \cdots, K)$, for each value of $x$, is the solution of the lower level problem:

$$
\left\{\begin{array}{l}
\min _{y_{i} \in Y_{i}} f_{i}\left(x, y_{i}\right)=c_{i} x+e_{i} y_{i} \\
\text { subject to : } A_{i} x+C_{i} y_{i} \leq b_{i}
\end{array}\right.
$$

where $c \in R^{n}, c_{i} \in R^{n}, d_{i} \in R^{m_{i}}, \quad e_{i} \in R^{m_{i}}, b \in R^{p}, \quad b_{i} \in R^{q_{i}}, A \in R^{p \times n}, \quad B_{i} \in R^{p \times m_{i}}$, $A_{i} \in R^{q_{i} \times n}, C_{i} \in R^{q_{i} \times m_{i}}, i=1,2, \ldots, K$.

\section{$S_{2}$ Model for BLMF decision problems}

For $\quad x \in X \subset R^{n}, \quad y_{i} \in Y_{i} \subset R^{m_{i}}, \quad Y=\left(Y_{1}, Y_{2}, \cdots, Y_{k}\right)^{T}, \quad F: X \times Y_{1} \times \ldots \times Y_{K} \rightarrow R^{1}$, $f_{i}: X \times Y_{i} \rightarrow R^{1}$, and $i=1,2, \ldots, K$, a linear BLMF decision problem in which $K(\geq 2)$ followers are involved and there are shared decision variables, objective functions and constraint functions among them is defined as follows.

It consists of finding a solution to the upper level problem 


$$
\left\{\begin{array}{c}
\min _{x \in X} F\left(x, y_{1}, \cdots, y_{K}\right)=c x+\sum_{s=1}^{K} d_{s} y_{s} \\
\text { subject to: } A x+\sum_{t=1}^{K} B_{t} y_{t} \leq b
\end{array}\right.
$$

where $y_{i}(i=1,2, \cdots, K)$, for each value of $x$, is the solution of the lower level problem:

$$
\left\{\begin{array}{c}
\min _{y_{1} \in Y_{1}, \ldots, y_{K} \in Y_{K}} f\left(x, y_{1}, \cdots, y_{K}\right)=c^{\prime} x+\sum_{j=1}^{K} e_{j} y_{j} \\
\text { subject to: } A^{\prime} x+\sum_{j=1}^{K} C_{j} y_{j} \leq b^{\prime}
\end{array}\right.
$$

where $c \in R^{n}, \quad c^{\prime} \in R^{n}, \quad d_{i} \in R^{m_{i}}, \quad e_{i} \in R^{m_{i}}, b \in R^{p}, b^{\prime} \in R^{q}, A \in R^{p \times n}, \quad B_{i} \in R^{p \times m_{i}}$, $A^{\prime} \in R^{q \times n}, C_{i} \in R^{q \times m_{i}}, i=1,2, \ldots, K$.

\section{$S_{3}$ Model for BLMF decision problems}

For $\quad x \in X \subset R^{n}, \quad y_{i} \in Y_{i} \subset R^{m_{i}}, \quad Y=\left(Y_{1}, Y_{2}, \cdots, Y_{k}\right)^{T}, \quad F: X \times Y_{1} \times \ldots \times Y_{K} \rightarrow R^{1}$, $f_{i}: X \times Y_{i} \rightarrow R^{1}$, and $i=1,2, \ldots, K$, a linear BLMF decision problem in which $K(\geq 2)$ followers are involved and there are shared decision variables and objective functions but separate constraint functions among them is defined as follows.

It consists of finding a solution to the upper level problem

$$
\left\{\begin{array}{c}
\min _{x \in X} F\left(x, y_{1}, \cdots, y_{K}\right)=c x+\sum_{s=1}^{K} d_{s} y_{s} \\
\text { subject to : } A x+\sum_{t=1}^{K} B_{t} y_{t} \leq b
\end{array}\right.
$$

where $y_{i}(i=1,2, \cdots, K)$, for each value of $x$, is the solution of the lower level problem: 


$$
\left\{\begin{array}{c}
\min _{y_{1} \in Y_{1}, \ldots, y_{K} \in Y_{K}} f\left(x, y_{1}, \cdots, y_{K}\right)=c^{\prime} x+\sum_{j=1}^{K} e_{j} y_{j} \\
\text { subject to : } A_{i} x+\sum_{j=1}^{K} C_{i j} y_{j} \leq b_{i}
\end{array}\right.
$$

where $c \in R^{n}, \quad c^{\prime} \in R^{n}, \quad d_{i} \in R^{m_{i}}, \quad e_{i} \in R^{m_{i}}, b \in R^{p}, b_{i} \in R^{q_{i}}, \quad A \in R^{p \times n}, \quad B_{i} \in R^{p \times m_{i}}$, $A_{i} \in R^{q_{i} \times n}, C_{i j} \in R^{q_{i} \times m_{j}}, i, j=1,2, \ldots, K$.

\section{$S_{4}$ Model for BLMF decision problems}

For $\quad x \in X \subset R^{n}, \quad y_{i} \in Y_{i} \subset R^{m_{i}}, \quad Y=\left(Y_{1}, Y_{2}, \cdots, Y_{k}\right)^{T}, \quad F: X \times Y_{1} \times \ldots \times Y_{K} \rightarrow R^{1}$, $f_{i}: X \times Y_{i} \rightarrow R^{1}$, and $i=1,2, \ldots, K$, a linear BLMF decision problem in which $K(\geq 2)$

followers are involved and there are shared decision variables and constraint functions but separate objective functions among them is defined as follows.

It consists of finding a solution to the upper level problem

$$
\left\{\begin{array}{c}
\min _{x \in X} F\left(x, y_{1}, \cdots, y_{K}\right)=c x+\sum_{s=1}^{K} d_{s} y_{s} \\
\text { subject to : } A x+\sum_{t=1}^{K} B_{t} y_{t} \leq b
\end{array}\right.
$$

where $y_{i}(i=1,2, \cdots, K)$, for each value of $x$, is the solution of the lower level problem:

$$
\left\{\begin{array}{c}
\min _{y_{1} \in Y_{1}, \ldots, y_{K} \in Y_{K}} f_{i}\left(x, y_{1}, \cdots, y_{K}\right)=c_{i} x+\sum_{j=1}^{K} e_{i j} y_{j} \\
\text { subject to : } A^{\prime} x+\sum_{j=1}^{K} C_{j} y_{j} \leq b^{\prime}
\end{array}\right.
$$

where $c \in R^{n}, \quad c_{i} \in R^{n}, \quad d_{i} \in R^{m_{i}}, \quad e_{i j} \in R^{m_{j}}, b \in R^{p}, b^{\prime} \in R^{q}, A \in R^{p \times n}, \quad B_{i} \in R^{p \times m_{i}}$, $A^{\prime} \in R^{q \times n}, C_{i} \in R^{q \times m_{i}}, i, j=1,2, \ldots, K$ 


\section{$S_{5}$ Model for BLMF decision problems}

For $\quad x \in X \subset R^{n}, \quad y_{i} \in Y_{i} \subset R^{m_{i}}, \quad Y=\left(Y_{1}, Y_{2}, \cdots, Y_{k}\right)^{T}, \quad F: X \times Y_{1} \times \ldots \times Y_{K} \rightarrow R^{1}$, $f_{i}: X \times Y_{i} \rightarrow R^{1}$, and $i=1,2, \ldots, K$, a linear BLMF decision problem in which $K(\geq 2)$

followers are involved and there are shared decision variables but separate objective and constraint functions among them is defined as follows.

It consists of finding a solution to the upper level problem

$$
\left\{\begin{array}{c}
\min _{x \in X} F\left(x, y_{1}, \cdots, y_{K}\right)=c x+\sum_{s=1}^{K} d_{s} y_{s} \\
\text { subject to: } A x+\sum_{t=1}^{K} B_{t} y_{t} \leq b
\end{array}\right.
$$

where $y_{i}(i=1,2, \cdots, K)$, for each value of $x$, is the solution of the lower level problem:

$$
\left\{\begin{array}{c}
\min _{y_{1} \in Y_{1}, \ldots, y_{K} \in Y_{K}} f_{i}\left(x, y_{1}, \cdots, y_{K}\right)=c_{i} x+\sum_{j=1}^{K} e_{i j} y_{j} \\
\text { subject to : } \quad A_{i} x+\sum_{j=1}^{K} C_{i j} y_{j} \leq b_{i}
\end{array}\right.
$$

where $c \in R^{n}, \quad c_{i} \in R^{n}, \quad d_{i} \in R^{m_{i}}, \quad e_{i j} \in R^{m_{j}}, b \in R^{p}, b_{i} \in R^{q_{i}}, \quad A \in R^{p \times n}, \quad B_{i} \in R^{p \times m_{i}}$, $A_{i} \in R^{q_{i} \times n}, C_{i j} \in R^{q_{i} \times m_{j}}, i, j=1,2, \ldots, K$.

\section{$S_{6}$ Model for BLMF decision problems}

For $\quad x \in X \subset R^{n}, \quad y_{i} \in Y_{i} \subset R^{m_{i}}, \quad Y=\left(Y_{1}, Y_{2}, \cdots, Y_{k}\right)^{T}, \quad z \in Z \subset R^{m}$, $F: X \times Y_{1} \times \ldots \times Y_{K} \times Z \rightarrow R^{1}$, and $f: X \times Y_{1} \times \ldots \times Y_{K} \times Z \rightarrow R^{1}, \quad i=1,2, \ldots, K$, a linear BLMF decision problem in which $K(\geq 2)$ followers are involved, there are shared 
objective functions, constraint functions and partial decision variables among them is defined as follows.

$$
\left\{\begin{array}{c}
\min _{x \in X} \quad F\left(x, y_{1}, \ldots, y_{K}, z\right)=c x+\sum_{s=1}^{K} d_{s} y_{s}+d z \\
\text { subject to : } A x+\sum_{t=1}^{K} B_{t} y_{t}+B z \leq b
\end{array}\right.
$$

where $y_{i}(i=1,2, \cdots, K)$ and $z$, for each value of $x$, are the solution of the lower level problem:

$$
\left\{\begin{array}{c}
\min _{y_{i} \in Y_{i}, z \in Z} f\left(x, y_{1}, \ldots, y_{K}, z\right)=c^{\prime} x+\sum_{j=1}^{K} e_{j} y_{j}+e z \\
\text { subject to : } A^{\prime} x+\sum_{j=1}^{K} C_{j} y_{j}+C^{\prime} z \leq b^{\prime}
\end{array}\right.
$$

where $c \in R^{n}, c^{\prime} \in R^{n}, d_{i} \in R^{m_{i}}, d \in R^{m}, e_{i} \in R^{m_{j}}, e \in R^{m}, b \in R^{p}, b^{\prime} \in R^{q}, A \in R^{p \times n}$, $B_{i} \in R^{p \times m_{i}}, B \in R^{p \times m}, A^{\prime} \in R^{q \times n}, C_{i} \in R^{q \times m_{i}}, C^{\prime} \in R^{q \times m}, i=1,2, \ldots, K$.

\section{$S_{7}$ Model for BLMF decision problems}

For $\quad x \in X \subset R^{n}, \quad y_{i} \in Y_{i} \subset R^{m_{i}}, \quad Y=\left(Y_{1}, Y_{2}, \cdots, Y_{k}\right)^{T}, \quad z \in Z \subset R^{m}$, $F: X \times Y_{1} \times \ldots \times Y_{K} \times Z \rightarrow R^{1}$, and $f: X \times Y_{1} \times \ldots \times Y_{K} \times Z \rightarrow R^{1}, \quad i=1,2, \ldots, K$, a linear BLMF decision problem in which $K(\geq 2)$ followers are involved and there are shared objective functions and partial decision variables but separate constraint functions among them is defined as follows.

$$
\left\{\begin{array}{c}
\min _{x \in X} \quad F\left(x, y_{1}, \ldots, y_{K}, z\right)=c x+\sum_{s=1}^{K} d_{s} y_{s}+d z \\
\text { subject to : } A x+\sum_{t=1}^{K} B_{t} y_{t}+B z \leq b
\end{array}\right.
$$


where $y_{i}(i=1,2, \cdots, K)$ and $z$, for each value of $x$, are the solution of the lower level problem:

$$
\left\{\begin{array}{c}
\min _{y_{i} \in Y_{i}, z \in Z} f\left(x, y_{1}, \ldots, y_{K}, z\right)=c^{\prime} x+\sum_{j=1}^{K} e_{j} y_{j}+e z \\
\text { subject to : } A_{i} x+\sum_{j=1}^{K} C_{i j} y_{j}+C_{i} z \leq b_{i}
\end{array}\right.
$$

where $c \in R^{n}, c^{\prime} \in R^{n}, d_{i} \in R^{m_{i}}, d \in R^{m}, e_{i} \in R^{m_{j}}, e \in R^{m}, b \in R^{p}, b_{i} \in R^{q_{i}}, A \in R^{p \times n}$, $B_{i} \in R^{p \times m_{i}}, B \in R^{p \times m}, A_{i} \in R^{q_{i} \times n}, C_{i j} \in R^{q_{i} \times m_{j}}, C_{i} \in R^{q_{i} \times m}, i, j=1,2, \ldots, K$.

\section{$S_{8}$ Model for BLMF decision problems}

For $\quad x \in X \subset R^{n}, \quad y_{i} \in Y_{i} \subset R^{m_{i}}, \quad Y=\left(Y_{1}, Y_{2}, \cdots, Y_{k}\right)^{T}, \quad z \in Z \subset R^{m}$, $F: X \times Y_{1} \times \ldots \times Y_{K} \times Z \rightarrow R^{1}$, and $f: X \times Y_{1} \times \ldots \times Y_{K} \times Z \rightarrow R^{1}, \quad i=1,2, \ldots, K$, a linear BLMF decision problem in which $K(\geq 2)$ followers are involved and there are shared constraint functions and partial decision variables but separate objective functions among them is defined as follows.

$$
\left\{\begin{array}{cc}
\min _{x \in X} \quad F\left(x, y_{1}, \ldots, y_{K}, z\right)=c x+\sum_{s=1}^{K} d_{s} y_{s}+d z \\
\text { subject to : } A x+\sum_{t=1}^{K} B_{t} y_{t}+B z \leq b
\end{array}\right.
$$

where $y_{i}(i=1,2, \cdots, K)$ and $z$, for each value of $x$, are the solution of the lower level problem: 


$$
\left\{\begin{array}{c}
\min _{y_{i} \in Y_{i}, z \in Z} f_{i}\left(x, y_{1}, \ldots, y_{K}, z\right)=c_{i} x+\sum_{j=1}^{K} e_{i j} y_{j}+e_{i} z \\
\text { subject to: } A^{\prime} x+\sum_{j=1}^{K} C_{j} y_{j}+C^{\prime} z \leq b^{\prime}
\end{array}\right.
$$

where $c \in R^{n}, c_{i} \in R^{n}, d_{i} \in R^{m_{i}}, d \in R^{m}, e_{i j} \in R^{m_{j}}, e \in R^{m}, b \in R^{p}, b^{\prime} \in R^{q}, A \in R^{p \times n}$, $B_{i} \in R^{p \times m_{i}}, B \in R^{p \times m}, A^{\prime} \in R^{q \times n}, C_{i} \in R^{q \times m_{i}}, C^{\prime} \in R^{q \times m}, i, j=1,2, \ldots, K$.

\section{$S_{9}$ Model for BLMF decision problems}

For $\quad x \in X \subset R^{n}, \quad y_{i} \in Y_{i} \subset R^{m_{i}}, \quad Y=\left(Y_{1}, Y_{2}, \cdots, Y_{k}\right)^{T}, \quad z \in Z \subset R^{m}$, $F: X \times Y_{1} \times \ldots \times Y_{K} \times Z \rightarrow R^{1}$, and $f: X \times Y_{1} \times \ldots \times Y_{K} \times Z \rightarrow R^{1}, \quad i=1,2, \ldots, K$, a linear BLMF decision problem in which $K(\geq 2)$ followers are involved and there are partial shared decision variables but separate objective functions and constraint functions among them is defined as follows.

$$
\left\{\begin{array}{c}
\min _{x \in X} \quad F\left(x, y_{1}, \ldots, y_{K}, z\right)=c x+\sum_{s=1}^{K} d_{s} y_{s}+d z \\
\text { subject to : } A x+\sum_{t=1}^{K} B_{t} y_{t}+B z \leq b
\end{array}\right.
$$

where $y_{i}(i=1,2, \cdots, K)$ and $z$, for each value of $x$, are the solution of the lower level problem:

$$
\left\{\begin{array}{c}
\min _{y_{i} \in Y_{i}, z \in Z} f_{i}\left(x, y_{1}, \ldots, y_{K}, z\right)=c_{i} x+\sum_{j=1}^{K} e_{i j} y_{j}+e_{i} z \\
\text { subject to : } A_{i} x+\sum_{j=1}^{K} C_{i j} y_{j}+C_{i} z \leq b_{i}
\end{array}\right.
$$


where $c \in R^{n}, c_{i} \in R^{n}, d_{i} \in R^{m_{i}}, d \in R^{m}, e_{i j} \in R^{m_{j}}, e_{i} \in R^{m}, b \in R^{p}, b_{i} \in R^{q_{i}}, A \in R^{p \times n}$, $B_{i} \in R^{p \times m_{i}}, B \in R^{p \times m}, A_{i} \in R^{q_{i} \times n}, C_{i j} \in R^{q_{i} \times m_{j}}, C^{\prime} \in R^{q \times m}, i, j=1,2, \ldots, K$.

The nine BLMF decision models define respectively the nine kinds of relationships among the followers described in the framework of BLMF decision problem. Obviously, these models will require their individual definitions for an optimal solution. Therefore, different approaches to derive an optimal solution for these models need to be developed. In particular, there are obvious differences between the uncooperative decision model $\left(S_{1}\right)$ of which there are no shared decision variables and cooperative model $\left(S_{2}-S_{5}\right)$ of which there are shared decision variables among the followers. In an uncooperative decision situation, the followers' reactions for each possible action of the leader can be determined by considering multiple individual optimizations respectively. While in a cooperative situation, the followers' reactions will be determined by dealing with a multi-objective optimization problem put forward by all the followers.

However, for any of the nine models, it is assumed that the leader knows completely the objective functions and constraints of these followers and the relationships among these objective and constraint functions. The control for decision variables is partitioned between the leader and the followers. Both the leader and the followers seek to minimize their individual payoff objective functions. The leader must anticipate all possible responses of followers based on their relationships.

\section{An optimal Solution for the Uncooperative Bilevel Multi-follower Decision Problem}


The uncooperative situation $\left(S_{1}\right)$ is the most basic form for BLMF decision problems over the nine kinds of decision models. This section, therefore, focuses on this model by giving the definition for an optimal solution and related theorems for solving the $S_{l}$ decision model.

Definition 1 A topological space is compact if every open cover of the entire space has a finite subcover. For example, $[a, b]$ is compact in $R$ (the Heine-Borel theorem) [12].

\section{Definition 2}

(a) Constraint region of a linear BLMF programming problem:

$S=\left\{\left(x, y_{1}, \ldots, y_{K}\right) \in X \times Y_{1} \times \ldots \times Y_{k}, A x+\sum_{t=1}^{K} B_{t} y_{t} \leq b\right.$,

$\left.A_{i} x+C_{i} y_{i} \leq b_{i}, i=1,2, \ldots, K\right\}$.

The linear BLFMP problem constraint region refers to all possible combinations of choices that the leader and followers may make.

(b) Projection of $S$ onto the leader's decision space:

$S(X)=\left\{x \in X: \exists y_{i} \in Y_{i}, A x+\sum_{t=1}^{K} B_{t} y_{t} \leq b, A_{i} x+C_{i} y_{i} \leq b_{i}, i=1,2, \ldots, K\right\}$.

Unlike the rules in an uncooperative game theory of which each player must choose a strategy simultaneously, the definition of the BLMF model requires that the leader move first by selecting an $x$ in attempt to minimize his/her objective subjecting to constraints of both upper and each lower level.

(c) Feasible set for each follower $\forall x \in S(X)$ : 
$S_{i}(x)=\left\{y_{i} \in Y_{i}:\left(x, y_{1}, \ldots, y_{K}\right) \in S\right\}, i=1,2, \ldots, K$

The feasible region for the follower is affected by the leader's choice of $x$, and allowable choices of each follower are the elements of $S$.

(d) Each follower's rational reaction set for $x \in S(X)$ :

$P_{i}(x)=\left\{y_{i} \in Y_{i}: y_{i} \in \arg \min \left[f_{i}\left(x, \hat{y}_{i}\right): \hat{y}_{i} \in S_{i}(x)\right]\right\}, i=1,2, \ldots, K$,

where $\arg \min \left[f_{i}\left(x, \hat{y}_{i}\right): \hat{y}_{i} \in S_{i}(x)\right]=\left\{y_{i} \in S_{i}(x): f_{i}\left(x, y_{i}\right) \leq f_{i}\left(x, \hat{y}_{i}\right), \hat{y}_{i} \in S_{i}(x)\right\}$.

The followers observe the leader's action and simultaneously react by selecting $y_{i}$ from his/her feasible set to minimize his/her objective function.

(e) Inducible region:

$I R=\left\{\left(x, y_{1}, \ldots, y_{K}\right):\left(x, y_{1}, \ldots, y_{K}\right) \in S, y_{i} \in P_{i}(x), i=1,2, \ldots, K\right\}$

Thus in terms of the above notations, the linear BLMF problem can be written as $\min \left\{F\left(x, y_{1}, \ldots, y_{K}\right):\left(x, y_{1}, \ldots, y_{K}\right) \in I R\right\}$

We present the following theorem to characterize the condition under which there is an optimal solution for a linear BLMF problem.

Theorem 1 If $S$ is nonempty and compact, there exists an optimal solution for a linear BLMF problem.

Proof: Since $S$ is nonempty, there exists a point $\left(x^{*}, y_{1}^{*}, \ldots, y_{K}^{*}\right) \in S$. Then, we have

$$
x^{*} \in S(X) \neq \phi
$$


by Definition 2b. Consequently, we have

$$
S_{i}\left(x^{*}\right) \neq \phi, \quad i=1,2, \ldots, K,
$$

by Definition 2 c. Because $S$ is compact and Definition 2 d, we have

$$
\begin{aligned}
P_{i}\left(x^{*}\right) & =\left\{y_{i} \in Y_{i}: y_{i} \in \arg \min \left[f_{i}\left(x^{*}, \hat{y}_{i}\right): \hat{y}_{i} \in S_{i}\left(x^{*}\right)\right]\right\} \\
& =\left\{y_{i} \in Y_{i}: y_{i} \in\left\{y_{i} \in S_{i}\left(x^{*}\right): f_{i}\left(x^{*}, y_{i}\right) \leq f_{i}\left(x^{*}, \hat{y}_{i}\right), \hat{y}_{i} \in S_{i}\left(x^{*}\right)\right\}\right\} \neq \phi,
\end{aligned}
$$

where $i=1,2, \ldots, K$. Hence, there exists $y_{i}^{0} \in P_{i}\left(x^{*}\right), i=1,2, \ldots, K$ such that

$$
\left(x^{*}, y_{1}^{0}, \ldots, y_{K}^{0}\right) \in S \text {. }
$$

Therefore, we have

$$
I R=\left\{\left(x, y_{1}, \ldots, y_{K}\right):\left(x, y_{1}, \ldots, y_{K}\right) \in S, y_{i} \in P_{i}(x), i=1,2, \ldots, K\right\} \neq \phi,
$$

by Definition 2e. Because we are minimizing a linear function

$\min _{x \in X} F\left(x, y_{1}, \ldots, y_{K}\right)=c x+\sum_{s=1}^{K} d_{s} y_{s}$ over $I R$, which is nonempty and bounded, an optimal

solution to the linear BLMF problem must exist. The proof is completed.

\section{An Extended Kuhn-Tucker Approach for the Uncooperative Bilevel Multi- follower Decision Problem}

The fundamental idea to deal with the uncooperative BLMF decision problems is that it replaces each follower's problem with its Kuhn-Tucker conditions and appends the resultant system to the leader's problem. The reformulation of the linear BLMF problem is a standard mathematical program and relatively easy to solve because all but complementary constraints are linear. Omitting or relaxing the constraints leaves a standard linear program that can be solved by using a simplex method [4]. In an uncooperative 
situation, the leader will be required to first select an $x \in X \subset R^{n}$ in attempting to minimize his/her objective subject to constraints of both the upper and each follower at the lower level. It then defines each follower's rational reaction set simultaneously by selecting the individual variable $y_{i} \in Y_{i} \subset R^{m_{i}}$ from his/her feasible set to minimize his/her objective function for the leader's choice. The Kuhn-Tucker approach is the most popular one for solving one leader one follower bilevel decision problems. Based on the definition of an optimal solution [11], an extended Kuhn-Tucker approach for the uncooperative BLMF decision problem is proven and described as follows.

Let us first consider a linear programming (LP) written as:

$\min f(x)=c x$

Subject to $A x \geq b$

$$
x \geq 0,
$$

where $c$ is an n-dimensional row vector, $b$ an m-dimensional column vector, $A$ an $m \times n$ matrix with $m \leq n$, and $x \in R^{n}$.

Let $\lambda \in R^{m}$ and $\mu \in R^{n}$ be the dual variables associated with constraints $A x \geq b$ and $x \geq 0$, respectively. Bard (Bard 1998) gave the following proposition.

Proposition 1 A necessary and sufficient condition that $\left(x^{*}\right)$ solves the above-mentioned LP is that there exist (row) vectors $\lambda^{*}, \mu^{*}$ such that $\left(x^{*}, \lambda^{*}, \mu^{*}\right)$ solves:

$$
\begin{aligned}
& \lambda A-\mu=-c \\
& A x-b \geq 0
\end{aligned}
$$




$$
\begin{aligned}
& \lambda(A x-b)=0 \\
& \mu x=0 \\
& x \geq 0, \lambda \geq 0, \mu \geq 0 .
\end{aligned}
$$

Proof: See (Bard 1998, PP. 59-60)

Let $u_{i} \in R^{p}, \quad v_{i} \in R^{q_{i}}$ and $w_{i} \in R^{m_{i}} \quad(i=1, \ldots, K)$ be the dual variables associated with constraints $\left(A x+\sum_{t=1}^{K} B_{t} y_{t} \leq b\right),\left(A_{i} x+C_{i} y_{i} \leq b_{i}, i=1,2, \ldots, K\right) \quad$ and $\quad y_{i} \geq 0 \quad(i=1, \ldots, K)$, respectively. We have the following theorem.

Theorem 2 A necessary and sufficient condition that $\left(x^{*}, y_{1}^{*}, \ldots, y_{K}^{*}\right)$ solves the linear BLMF problem $1 \mathrm{a}$ is that there exist (row) vectors $u_{1}^{*}, u_{2}^{*}, \ldots, u_{K}^{*}, v_{1}^{*}, v_{2}^{*}, \ldots, v_{K}^{*}$ and $w_{1}^{*}, w_{2}^{*}, \ldots, w_{K}^{*}$ such that $\left(x^{*}, y_{1}^{*}, \ldots, y_{K}^{*}, u_{1}^{*}, \ldots, u_{K}^{*}, v_{1}^{*}, \ldots, v_{K}^{*}, w_{1}^{*}, \ldots, w_{K}^{*}\right)$ solve:

$$
\begin{aligned}
& \min F\left(x, y_{1}, \ldots, y_{K}\right)=c x+\sum_{s=1}^{K} d_{s} y_{s} \\
& \text { subject to } A x+\sum_{t=1}^{k} B_{t} y_{t} \leq b \\
& A_{i} x+C_{i} y_{i} \leq b_{i} \\
& u_{i} B_{i}+v_{i} C_{i}-w_{i}=-e_{i} \\
& u_{i}\left(b-A x-\sum_{t=1}^{K} B_{t} y_{t}\right)+v_{i}\left(b_{i}-A_{i} x-C_{i} y_{i}\right)+w_{i} y_{i}=0 \\
& x \geq 0, y_{i} \geq 0, u_{i} \geq 0, v_{i} \geq 0, w_{i} \geq 0, i=1,2, \ldots, K,
\end{aligned}
$$

where $i=1,2 \ldots, K$. 


\section{Proof:}

1). Let us get an explicit expression of (2).

Rewrite (2) as follows:

$$
\begin{aligned}
& \min F\left(x, y_{1}, \ldots, y_{K}\right) \\
& \text { subject to }\left(x, y_{1}, \ldots, y_{K}\right) \in I R .
\end{aligned}
$$

We have

$$
\begin{gathered}
\min F\left(x, y_{1}, \ldots, y_{K}\right) \\
\text { subject to }\left(x, y_{1}, \ldots, y_{K}\right) \in S \\
y_{i}=P_{i}(x),
\end{gathered}
$$

where $i=1,2, \ldots, K$, by Definition 2e. Then, we have

$$
\begin{aligned}
& \min F\left(x, y_{1}, \ldots, y_{K}\right) \\
& \text { subject to }\left(x, y_{1}, \ldots, y_{K}\right) \in S \\
& \qquad y_{i} \in \arg \min \left[f_{i}\left(x, \hat{y}_{i}\right): \hat{y}_{i} \in S_{i}(x)\right],
\end{aligned}
$$

where $i=1,2, \ldots, K$, by Definition $2 \mathrm{~d}$. We rewrite it as:

$$
\begin{aligned}
& \min F\left(x, y_{1}, \ldots, y_{K}\right) \\
& \text { subject to }\left(x, y_{1}, \ldots, y_{K}\right) \in S \\
& \\
& \min f_{i}\left(x, y_{i}\right) \\
& \text { subject to } y_{i} \in S_{i}(x),
\end{aligned}
$$

where $i=1,2, \ldots, K$. We have

$$
\min F\left(x, y_{1}, \ldots, y_{K}\right)
$$

subject to $\left(x, y_{1}, \ldots, y_{K}\right) \in S$ 


$$
\begin{aligned}
& \min _{y_{i} \in Y_{i}} f_{i}\left(x, y_{i}\right) \\
& \text { subject to }\left(x, y_{1}, \ldots, y_{K}\right) \in S,
\end{aligned}
$$

where $i=1,2, \ldots, K$, by Definition 2c. Consequently, we can have

$$
\begin{aligned}
& \min F\left(x, y_{1}, \ldots, y_{K}\right)=c x+\sum_{s=1}^{K} d_{s} y_{s} \\
& \text { subject to } A x+\sum_{t=1}^{K} B_{t} y_{t} \leq b \\
& \qquad A_{j} x+C_{j} y_{j} \leq b_{j}, j=1,2, \ldots, K \\
& \min _{y_{i} \in Y_{i}} f_{i}\left(x, y_{i}\right)=c_{i} x+e_{i} y_{i} \\
& \operatorname{subject} \text { to } A x+\sum_{t=1}^{K} B_{t} y_{t} \leq b \\
& A_{j} x+C_{j} y_{j} \leq b_{j}, j=1,2, \ldots, K,
\end{aligned}
$$

where $i=1,2, \ldots, K$, by Definition 2 a.

This simple transformation has shown that to solve the linear BLMF programming (1a) is equivalent to solve the problem (4a-f).

2). Necessity is obvious from (4a-f).

3). Sufficiency.

If $\left(x^{*}, y_{1}^{*}, \ldots, y_{K}^{*}\right)$ is an optimal solution of (1a), we need to show that there exist (row) vectors $u_{1}^{*}, u_{2}^{*}, \ldots, u_{K}^{*}, v_{1}^{*}, v_{2}^{*}, \ldots, v_{K}^{*}$ and $w_{1}^{*}, w_{2}^{*}, \ldots, w_{K}^{*} \operatorname{such}$ that $\left(x^{*}, y_{1}^{*}, \ldots, y_{K}^{*}, u_{1}^{*}, \ldots, u_{K}^{*}\right.$, $\left.v_{1}^{*}, \ldots, v_{K}^{*}, w_{1}^{*}, \ldots, w_{K}^{*}\right)$ solve $(3 \mathrm{a}-\mathrm{f})$. Going one step further, we only need to prove that there exist (row) vectors $u_{1}^{*}, u_{2}^{*}, \ldots, u_{K}^{*}, v_{1}^{*}, v_{2}^{*}, \ldots, v_{K}^{*}$ and $w_{1}^{*}, w_{2}^{*}, \ldots, w_{K}^{*}$ such that $\left(x^{*}, y_{1}^{*}, \ldots, y_{K}^{*}, u_{1}^{*}, \ldots, u_{K}^{*}, v_{1}^{*}, \ldots, v_{K}^{*}, w_{1}^{*}, \ldots, w_{K}^{*}\right)$ satisfies the following conditions 


$$
\begin{aligned}
& u_{i} B_{i}+v_{i} C_{i}-w_{i}=-e_{i} \\
& u_{i}\left(b-A x-\sum_{t=1}^{K} B_{t} y_{t}\right)=0 \\
& v_{i}\left(b_{i}-A_{i} x-C_{i} y_{i}\right)=0 \\
& w_{i} y_{i}=0
\end{aligned}
$$

where $u_{i} \in R^{p}, v_{i} \in R^{q_{i}}, \quad w_{i} \in R^{m_{i}}, i=1,2, \ldots, K$ and they are not negative variables.

Because $\left(x^{*}, y_{1}^{*}, \ldots, y_{K}^{*}\right)$ is an optimal solution of $(1 \mathrm{a})$, we have

$$
\left(x^{*}, y_{1}^{*}, \ldots, y_{K}^{*}\right) \in I R
$$

by (2). Thus we have

$$
y_{i}^{*} \in P_{i}\left(x^{*}\right)
$$

where $i=1,2, \ldots, K$, by Definition $2 \mathrm{e}$. Consequently, $\left(y_{1}^{*}, y_{2}^{*}, \ldots, y_{K}^{*}\right)$ is an optimal solution to the following problem:

$$
\min \left(f_{i}\left(x, y_{i}\right): y_{i} \in S_{i}\left(x^{*}\right)\right)
$$

where $i=1,2, \ldots, K$, by Definition 2d. Rewrite (6) as follows

$$
\begin{aligned}
& \min f\left(x, y_{i}\right) \\
& \text { subject to } y_{i} \in S_{i}(x) \\
& x=x^{*} \\
& y_{j}=y_{j}^{*}(j=1,2, \ldots, K, j \neq i),
\end{aligned}
$$

where $i=1,2, \ldots, K$. From Definition 2c, we have

$$
\begin{aligned}
& \min f_{i}\left(x, y_{i}\right)=c_{i} x+e_{i} y_{i} \\
& \text { subject to } A x+\sum_{t=1}^{K} B_{t} y_{t} \leq b
\end{aligned}
$$




$$
\begin{aligned}
& A_{j} x+C_{j} y_{j} \leq b_{j}, j=1,2, \ldots, K \\
& x=x^{*} \\
& y_{i} \geq 0 \\
& y_{j}=y_{j}^{*}, j=1,2, \ldots, K, j \neq i,
\end{aligned}
$$

where $i=1,2, \ldots, K$.

To simplify (7a-f), we can have

$$
\begin{aligned}
& \min f_{i}\left(x, y_{i}\right)=c_{i} x+e_{i} y_{i} \\
& \text { subject to } A x+\sum_{t=1}^{K} B_{t} y_{t} \leq b \\
& A_{i} x+C_{i} y_{i} \leq b_{i} \\
& x=x^{*} \\
& y_{i} \geq 0 \\
& y_{j}=y_{j}^{*}, j=1,2, \ldots, K, j \neq i,
\end{aligned}
$$

where $i=1,2, \ldots, K$. Thus simplifying (8a-f), we can have

$$
\begin{gathered}
\min f_{i}\left(y_{i}\right)=e_{i} y_{i} \\
\text { subject to }-\left(\begin{array}{l}
B_{i} \\
C_{i}
\end{array}\right) y_{i} \geq-\left(\begin{array}{l}
b-A x^{*}-\sum_{j=1, j \neq i}^{K} B_{j} y_{j}^{*} \\
b_{i}-A_{i} x^{*}
\end{array}\right) \\
y_{i} \geq 0
\end{gathered}
$$

where $i=1,2, \ldots, K$.

Now we see that $y_{i}^{*}$ is an optimal solution of $(9 \mathrm{a}-\mathrm{c})$ which is a LP problem. By Proposition 1 , there exist vectors $\lambda_{i}^{*}, \mu_{i}^{*}, i=1,2, \ldots, K$ that satisfy the following system 


$$
\begin{aligned}
& \lambda_{i}\left(\begin{array}{l}
B_{i} \\
C_{i}
\end{array}\right)-\mu_{i}=-e_{i} \\
& \lambda_{i}\left(-\left(\begin{array}{l}
B_{i} \\
C_{i}
\end{array}\right) y_{i}+\left(\begin{array}{l}
b-A x^{*}-\sum_{j=1, j \neq i}^{K} B_{j} y_{j}^{*} \\
b_{i}-A_{i} x^{*}
\end{array}\right)=0\right. \\
& \mu_{i} y_{i}=0
\end{aligned}
$$

where $\lambda_{i} \in R^{p+q_{i}}, \mu_{i} \in R^{m_{i}}, i=1,2, \ldots, K$.

Let $u_{i} \in R^{p}, v_{i} \in R^{q_{i}}, w_{i} \in R^{m_{i}}$ and define

$$
\begin{aligned}
& \lambda_{i}=\left(u_{i}, v_{i}\right) \\
& w_{i}=\mu_{i},
\end{aligned}
$$

where $i=1,2, \ldots, K$.

Thus we have $\left(x^{*}, y_{1}^{*}, \ldots, y_{K}^{*}, u_{1}^{*}, \ldots, u_{K}^{*}, v_{1}^{*}, \ldots, v_{K}^{*}, w_{1}^{*}, \ldots, w_{K}^{*}\right)$ that satisfy $(5 \mathrm{a}-\mathrm{d})$. Our proof is completed.

Theorem 2 indicates that the most direct approach for solving (1a) is to solve the equivalent mathematical program given in (3a-f). One of its advantages is that it allows a more robust model to be solved without introducing any new computational difficulties.

\section{A Real Case Study of a Road-Network Problem}

In general, BLMF decision actions have the following three features: (1) there exists decision units within a predominantly hierarchical structure; (2) each unit at the lower level executes its policies after, and in view of, decisions made at the upper level; (3) each unit independently optimizes its objective but is affected by the actions of other units. When a 
real-world BLMF decision model, which could be any kind of the specific situations shown in the proposed framework, is established, a suitable approach must be selected and used for solving the problem. This section shows an uncooperative BLMF decision problem of a road network with hypothetical problem parameters. The proposed extended Kuhn-Tucker approach is used for solving the problem.

A road network problem involves the improvement of the road network through capacity expansion, traffic signals synchronization or vehicle guidance systems. The road management committee (the leader) is assumed to control these decision variables. The committee's decision can influence directly or indirectly the travel choices of the road network users. There are two kinds of road network users (the followers), one is public traffic user group and another is private traffic users. Let $x=\left(x_{1}, x_{2}\right)$ denote the decision vector of the road management committee, $X$ the set of feasible decision variables, $y, z$ the decision vector (one element) of the two followers respectively, and $c_{i}\left(x_{1}, x_{2}, y, z\right)$ the travel delay along a link $i$. The road management committee's main objective is to minimize, over the set $X$, the system travel cost $\sum_{i} a_{i} c_{i}\left(x_{1}, x_{2}, y, z\right)=F\left(x_{1}, x_{2}, y, z\right)$. Both kinds of network users seek to minimize their travel delays $\min f_{1}\left(x_{1}, x_{2}, y\right)$ and $\min f_{2}\left(x_{1}, x_{2}, z\right)$ respectively. The committee also seeks its minimized travel delays. However, the committee is interested in minimizing total travel time for all kinds of users, while each user group only wants to optimize its own travel time. The committee has known the objectives and constraints of the two groups of road network users. The two groups of users have different objective functions and different constraints. This is a typical uncooperative BLMF decision problem. 
In order to easily show the application for the proposed extended Kuhn-Tucker approach, a road-network decision problem model is established by simplifying it into the following linear BLMF decision model:

$$
\begin{aligned}
& \min _{x_{1}, x_{2} \in X} F\left(x_{1}, x_{2}, y, z\right)=3 x_{1}+8 x_{2}+7 y+11 z \\
& \text { subject to } 5 x_{1}+2 x_{2}-y+6 z \leq 40 \\
& 6 x_{1}-x_{2}+13 y \leq 15 \\
& x_{1}+x_{2}-7 z \leq 10 \\
& 7 y+4 z \leq 20 \\
& \quad \min _{y \in Y} f_{1}\left(x_{1}, x_{2}, y\right)=2 x_{1}+x_{2}-y \\
& \quad \operatorname{subject~to~} 5 x_{1}+7 y \leq 15 \\
& \quad-4 x_{2}+25 y \leq 3 \\
& \min _{z \in Z} f_{2}\left(x_{1}, x_{2}, z\right)=15 x_{1}-x_{2}+80 z \\
& \text { subject to } 40 x_{1}+z \leq 5 .
\end{aligned}
$$

where $x_{1}, x_{2} \in R^{1}, \quad y \in R^{1}, \quad z \in R^{1}$ and $X=\left\{x_{1}>0, x_{2}>0\right\}, \quad Y=\{y>0\}, \quad Z=\{z>0\}$. According to the Kuhn-Tucker approach, let us write all the inequalities but $x>0$ for the model as follows:

$$
\begin{aligned}
& g_{u, 1}\left(x_{1}, x_{2}, y, z\right)=40-\left(5 x_{1}+2 x_{2}-y+6 z\right) \geq 0 \\
& g_{u, 2}\left(x_{1}, x_{2}, y, z\right)=15-\left(6 x_{1}-x_{2}+13 y\right) \geq 0 \\
& g_{u, 3}\left(x_{1}, x_{2}, y, z\right)=10-\left(x_{1}+x_{2}-7 z\right) \geq 0 \\
& g_{u, 4}\left(x_{1}, x_{2}, y, z\right)=20-(7 y+4 z) \geq 0
\end{aligned}
$$




$$
\begin{aligned}
& g_{v 1,1}\left(x_{1}, x_{2}, y, z\right)=15-\left(5 x_{1}+7 y\right) \geq 0 \\
& g_{v 1,2}\left(x_{1}, x_{2}, y, z\right)=3-\left(-4 x_{2}+25 y\right) \geq 0 \\
& g_{w 1,1}\left(x_{1}, x_{2}, y, z\right)=y \geq 0 \\
& g_{v 2,1}\left(x_{1}, x_{2}, y, z\right)=5-\left(40 x_{1}+z\right) \geq 0 \\
& g_{w 2,1}\left(x_{1}, x_{2}, y, z\right)=z \geq 0 .
\end{aligned}
$$

From (3a-f), we have

$$
\begin{aligned}
\min \left(3 x_{1}+\right. & \left.8 x_{2}+7 y+11 z\right) \\
\text { subject to } & 5 x_{1}+2 x_{2}-y+6 z \leq 40 \\
& 6 x_{1}-x_{2}+13 y \leq 15 \\
& x_{1}+x_{2}-7 z \leq 10 \\
& 7 y+4 z \leq 20 \\
& 5 x_{1}+7 y \leq 15 \\
& -4 x_{2}+25 y \leq 3 \\
& 40 x_{1}+z \leq 5 \\
& -u_{11}+13 u_{12}+7 u_{13}+7 v_{11}+25 v_{12}-w_{11}=1 \\
& 6 u_{21}-7 u_{22}+4 u_{23}+v_{21}-w_{21}=-80 \\
& g_{u, 1} u_{11}+g_{u, 2} u_{12}+g_{u, 4} u_{13}+g_{v 1,1} v_{11}+g_{v 1,2} v_{12}+g_{w 1,1} w_{11}=0 \\
& g_{u, 1} u_{21}+g_{u, 3} u_{22}+g_{u, 4} u_{23}+g_{v 2,1} v_{21}+g_{w 2,1} w_{21}=0 \\
& x_{1}>0, x_{2}>0, y>0, z>0 \\
& 0, u_{12} \geq 0, u_{13} \geq 0, v_{11} \geq 0, v_{12} \geq 0, w_{11} \geq 0 \\
& \\
& \\
& \\
& \\
&
\end{aligned}
$$


From (11i), (11j), (11m), (11n) and (11o), we can have following four possibilities.

Case 1: $\left(u_{11}, u_{12}, u_{13}, v_{11}, v_{12}, w_{11}, u_{21}, u_{22}, u_{23}, v_{21}, w_{21}\right)=(0,1 / 13,0,0,0,0,0,8 / 7,0,0,0)$

Case 2: $\left(u_{11}, u_{12}, u_{13}, v_{11}, v_{12}, w_{11}, u_{21}, u_{22}, u_{23}, v_{21}, w_{21}\right)=(0,0,1 / 7,0,0,0,0,8 / 7,0,0,0)$

Case 3: $\left(u_{11}, u_{12}, u_{13}, v_{11}, v_{12}, w_{11}, u_{21}, u_{22}, u_{23}, v_{21}, w_{21}\right)=(0,0,0,1 / 7,0,0,0,8 / 7,0,0,0)$

Case 4: $\left(u_{11}, u_{12}, u_{13}, v_{11}, v_{12}, w_{11}, u_{21}, u_{22}, u_{23}, v_{21}, w_{21}\right)=(0,0,0,0,1 / 25,0,0,8 / 7,0,0,0)$

From Case 1, (11k) and (111) we have

$$
\begin{aligned}
& g_{u, 2}\left(x_{1}, x_{2}, y, z\right)=15-\left(6 x_{1}-x_{2}+13 y\right)=0 \\
& g_{u, 3}\left(x_{1}, x_{2}, y, z\right)=10-\left(x_{1}+x_{2}-7 z\right)=0 .
\end{aligned}
$$

Consequently, (11) can be rewritten as follows:

$$
\begin{gathered}
\min \left(3 x_{1}+8 x_{2}+7 y+11 z\right) \\
\text { subject to } 5 x_{1}+2 x_{2}-y+6 z \leq 40 \\
6 x_{1}-x_{2}+13 y=15 \\
x_{1}+x_{2}-7 z=10 \\
7 y+4 z \leq 20 \\
5 x_{1}+7 y \leq 15 \\
-4 x_{2}+25 y \leq 3 \\
40 x_{1}+z \leq 5 \\
x_{1}>0, x_{2}>0, y>0, z>0
\end{gathered}
$$

Using the simplex method, we found that a solution occurs at the point $\left(x_{1}^{1}, x_{2}^{1}, y^{1}, z^{1}\right)=(0.12,11.79,2.01,0.27)$ with $F^{1}=111.69, f_{1}^{1}=10.02$ and $f_{2}^{1}=11.61$. 
By using the same way as that of Case 1, we found that it is infeasible for Cases 2 and 4, respectively; a solution occurs at the point $\left(x_{1}^{3}, x_{2}^{3}, y^{3}, z^{3}\right)=(0.12,12.48,2.06,0.37)$ with $F^{3}=118.66, f_{1}^{3}=10.66$ and $f_{2}^{3}=17.36$ for Case 3 . By examining the above procedure, we found that an optimal solution occurs at the point $\left(x_{1}^{*}, x_{2}^{*}, y^{*}, z^{*}\right)=(0.12,11.79,2.01,0.27)$ with $F^{*}=111.69, f_{1}^{*}=10.02$ and $f_{2}^{*}=11.61$.

The result shows that an optimal solution for the road management committee is to take the two decision variables as 0.12 and 11.79 through anticipating all possible responses of followers. Each follower is assumed to execute simultaneously his/her individual policies after decisions of the leader for the two decision variables. That is, the two followers will take values of their travel decision variables 2.01 and 0.27 respectively as their reaction for the committee. The two followers can thus reach an objective 10.02 and 11.61 respectively and the committee will get an objective of travel cost 111.69.

\section{Conclusions and Further Study}

Many organizations such as universities, Governmental departments, are decentralized, and their decision units are within an hierarchical structure. The execution of their decisions is often sequential and the leader's decision can be affected by the responses of his or her multiple followers. Therefore, BLMF decision-making is a common issue in organizational management. This paper has successfully established a framework for the BLMF decision problem which identifies nine kinds of relationships among the followers. These bilevel decision relationships may occur in different organizations' decision actions or within different decision actions of one organization. For each of the nine relationships, a 
corresponding BLMF decision model has been proposed. In particular, this paper has proposed related theories focusing on the uncooperative BLMF decision model $\left(S_{1}\right)$. An extended Kuhn-Tucker approach for solving the specific kind of BLMF decision problems is then developed. Finally, a real-case study of a road-network problem illustrates the application of the proposed BLMF decision technique. Further study will involve the development of approaches for the other eight BLMF models $\left(S_{2}\right.$ to $\left.S_{9}\right)$ as described in the

proposed framework. A decision support system will then need to be developed to implement the proposed techniques for solving all the nine kinds of BLMF decision problems.

\section{Acknowledgments}

The work presented in this paper was supported by Australian Research Council (ARC) under discovery grants DP0557154 and DP0559213.

\section{References}

[1] M.A. Amouzegar and K. Moshirvaziri, Strategic management decision support system: An analysis of the environmental policy issues, Environmental Modeling and Assessment, 6, 297-306 (2001).

[2] G. Anandalingam and T. Friesz, Hierarchical optimization: An introduction, Annals of Operations Research, 34, 1-11 (1992).

[3] J. Bard, Optimality conditions for the bilevel programming problem, Naval Research Logistics Quarterly, 31, 13-26 (1984).

[4] J. Bard, Practical Bilevel Optimization (Kluwer Academic Publishers, Dordrecht, The Netherlands, 1998).

[5] O. Ben-Ayed, Bilevel linear programming, Computers and Operations Research, 20, 485-501 (1993). 
[6] W. Bialas and M. Karwan, Two-level linear programming, Management Science, 30, 1004-1020 (1984).

[7] S. Dempe, Foundations of Bilevel Programming (Kluwer Academic Publishers, Dordrecht, The Netherlands, 2002).

[8] M. Labbé, P. Marcotte, and G. Savard, A bilevel model of taxation and its application to optimal highway pricing, Management Science, 44, 1608-1822 (1999).

[9] P. Marcotte, Network design with congestion effects: a case of bilevel programming, Mathematical Programming, 34, 142-162 (1986).

[10] C. Shi, G. Zhang, and J. Lu, On the definition of linear bilevel programming solution, Applied Mathematics and Computation, 160(1), 169-176 (2005).

[11] C. Shi, J. Lu, and G. Zhang, An extended Kuhn-Tucker approach for linear bilevel programming, Applied Mathematics and Computation, 162(1), 51-63 (2005).

[12] University of Cambridge (2001), http://thesaurus.maths.org/dictionary/map/word/10037 